\title{
Stress variations near surfaces in diamond-like amorphous carbon
}

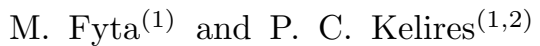 \\ (1) Physics Department, University of Crete, P.O. Box 2208, 71003 Heraclion, Crete, Greece \\ ${ }^{(2)}$ Foundation for Research and Technology-Hellas (FORTH), \\ P.O. Box 1527, 711 10, Heraklion, Crete, Greece.
}

\begin{abstract}
Using Monte Carlo simulations within the empirical potential approach, we examine the effect produced by the surface environment on the atomic level stresses in tetrahedral amorphous carbon. Both the distribution of stresses and the distributions of $\mathrm{sp}^{2}$ and $\mathrm{sp}^{3}$ atoms as a function of depth from the surface are highly inhomogeneous. They show the same close relationship between local stress and bonding hybridization found previously in the bulk of the material. Compressive local stress favors the formation of $\mathrm{sp}^{3}$ sites, while tensile stress favors the formation of $\mathrm{sp}^{2}$ sites.
\end{abstract}

\section{INTRODUCTION}

One of the important properties of diamond-like tetrahedral amorphous carbon (ta-C), is believed to be its possession of high average compressive stress. The presence of such intrinsic stress is desirable, in one aspect, because it is associated with a high percentage of tetrahedral $\left(\mathrm{sp}^{3}\right)$ atoms. However, it is also desirable to relieve the compressive stress because is believed to produce adhesion failure at the film/substrate interface. The origin of this stress and its relationship to the formation of $\mathrm{sp}^{3}$ sites is still a matter of debate [1]. One view is that the compressive stress is produced by the energy of ion bombardment in the deposition process, and that it is responsible for a thermodynamic phase transition from $\mathrm{sp}^{2} \rightarrow \mathrm{sp}^{3}$ phase at a critical average value [2]. A model to explain the dependence of compressive stress on ion energy has been developed [3]. Another view is that "subplantation" events (i.e., shallow implantation of incoming atoms) during deposition produce densification, and as a result this promotes the $\mathrm{sp}^{3}$ bonding and the generation of compressive stress [4].

An alternative approach to this issue is based on the concept of atomic level stresses [5]. The logic behind it rests on the observation that stress generation is a local process extending over few atomic volumes, and that what really matters is the local character of the stress tensor. It was shown that the average compressive stress is not a crucial factor for the stabilization of $\mathrm{sp}^{3}$ sites, as it could be negligibly small [5]. Note that current experimental efforts to solve the adhesion problem [ 6, 7] aim to relieve the average stress through post-growth thermal annealing. Instead, it is proposed that the compressive local stresses are the crucial parameter. In this approach, bonding hybridization is closely associated with the local stress conditions: compressive stresses favor the formation of $\mathrm{sp}^{3} \mathrm{sites}$, while tensile conditions favor $\mathrm{sp}^{2}$ sites. This correspondence was derived from considerations of the bulk material.

In this paper, we take into account the surface environment and examine how global this relationship is. By analyzing consistently the variations of stresses and the distributions of atoms as functions of depth, we unambigiously show the validity of the local stress - bonding hybridization relationship in ta-C. In the following, we first outline the method used in our calculations, and then we give the results regarding the surface microstructure and atomic stresses, and discuss their association.

\section{THEORETICAL METHODS}

The investigations are based on Monte Carlo (MC) simulations within the empirical potential approach 5]. We use slab supercells with two free surfaces, and with periodic boundary conditions in the two lateral dimensions. The cells are deep enough $(\sim 30 \AA$ in each direction) to sufficiently describe the variations with depth. They contain 720 atoms.

The amorphous networks are generated by quenching the liquid under pressure within the constant pressuretemperature $(\mathrm{N}, \mathrm{P}, \mathrm{T})$ ensemble. The liquid is equilibrated at $\sim 9000 \mathrm{~K}$ and then cooled to $300 \mathrm{~K}$ under various pressures up to $300 \mathrm{GPa}$ and at cooling rates up to $\sim 25$ (MC steps)/atom-K. Alternatively, one can use the constantvolume $(\mathrm{N}, \mathrm{V}, \mathrm{T})$ ensemble and reflecting walls at the two surfaces to contain the liquid while quenching. After removing the pressure (or the walls), the cell density is equilibrated and the atomic positions are thoroughly relaxed.

The empirical potential of Tersoff [ [] is used to model the interactions. It is extensively tested and found to describe reasonably well the properties of ta-C [5, 9]. The potential describes strain quite well [10]. Though in principle less accurate than ab initio methods, this approach permits us to extract the atomic level stresses, which are otherwise inaccessible. The atomic stresses are in general the result of local incompatibilities, usually due to atomic 
size mismatch. They are defined [5] by considering an atomic compression (tension)

$$
\sigma_{i}=-d E_{i} / d \ln V \sim p_{i} \Omega_{i}
$$

where $E_{i}$ is the energy of atom $i$ and $V$ is the volume. Note that the decomposition of total energy into atomic contributions is not possible using first-principles methods. Dividing by the atomic volume $\Omega_{i}$ converts $\sigma_{i}$ into units of pressure $p_{i}$. This local hydrostatic pressure describes the local density fluctuations. The total intrinsic stress of the system can be calculated by summing up the $\sigma_{i}$ over all atoms. For a completely strain compensated system the total stress is zero. This means that the individual contributions cancel each other, but it does not mean that themselves are also diminished.

\section{RESULTS}

\section{A. Structure}

We first briefly describe the surface structure of ta-C which has been simulated with the same MC methodology earlier [11]. It was found that the amorphous surface layer reconstructs to form distorted sixfold-ring patterns of $\mathrm{sp}^{2}$ sites (along with odd-membered rings and fourfold atoms) in a graphite-like manner. This is illustrated here in Fig. 1 , showing the structure of one of the two surfaces in a typical slab cell with average coordination $\bar{z} \simeq 3.75$. It seems that this structure is a generic feature of ta-C, supported also by other recent simulations 12]. This specific feature of the surface layer has not yet been identified by experiment, although the enhancement with $\mathrm{sp}^{2}$ sites is established 13. The graphite-like nature of the surface in ta-C is in sharp contrast to its bulk topology, for which researchers have reached a consensus about the clustering of $\mathrm{sp}^{2}$ sites: they are found in pairs, chains and small clusters rather than in aromatic rings.

It is essential for our purposes to have a detailed picture of how $\mathrm{sp}^{2}$ and $\mathrm{sp}^{3}$ atoms are distributed in the slab cells as a function of distance $\mathrm{z}$ from the two surfaces. We thus compute the probabilities (unnormalized) of finding threefold and fourfold atoms at depth $\mathrm{z}$ in the slab cell of Fig. 1. These are defined formally as the atomic position densities of states $P(z)=d N / d z$, where $d N$ is the number of $\mathrm{sp}^{2}$ or of $\mathrm{sp}^{3}$ sites lying in the vertical position interval between $z$ and $z+d z$. The resulting distributions are shown in Fig. 2. There are two prominent characteristics. The probability for $\mathrm{sp}^{2}$ atoms at the surface layers ( $\sim 2 \AA$ thickness) is pronounced in accordance with Fig. 1, while the probability for $\mathrm{sp}^{3}$ atoms in this layer is small. On the other hand, at the middle of the slab where bulklike conditions dominate, the probability for $\mathrm{sp}^{3}$ atoms is maximized while for $\mathrm{sp}^{2}$ atoms is minimized. In general, there is an overall tendency through the whole slab to have enhancement of the probability for one type of coordination in a certain region, and at the same time reduction of the probability for the other type in this region.

\section{B. Atomic level stresses}

Before addressing the dependence of local stresses on depth, we examine their average distribution. As shown in Ref. [5], insight into the stress field is provided by calculating the probability distributions (stress density of states) over all atoms in the system. We used the same analysis here for the cell discussed above. The results are given in Fig. 3. $P(\sigma)$ is the unnormalized probability of finding an atom under local stress $\sigma$; positive values indicate compressive stress. We show the decomposed distributions according to coordination. The central conclusion from this analysis is that all features observed previously in the bulk system are still valid, despite the presence of large discontinuities introduced by the surface. These features are: (a) The majority of $\mathrm{sp}^{3}$ atoms are under compressive stress with a peak (most probable) value around $\sim 7 \mathrm{GPa}$. On the other hand, the majority of $\mathrm{sp}^{2}$ atoms are under tensile stress with a larger peak value around $\sim 20 \mathrm{GPa}$. This shows a tendency of the fewer $\mathrm{sp}^{2}$ atoms to compensate the compressive stress of the $\mathrm{sp}^{3}$ atoms. (b) Both distributions are broad covering compressive and tensile values, so fourfold (threefold) atoms could be under tension (compression) as well, although less likely. Thus, an inhomogeneous stress distribution arises in the network.

The stress inhomogeneity has another component in the present case: the surface effect. To show quantitatively how the atomic stresses vary as a function of depth, we compute the probability of finding a threefold (fourfold) atom under tensile or compressive stress at depth z. We plot in Fig. 4 the probabilities for threefold and fourfold atoms to have tensile and compressive stress, respectively. These are the dominant stress conditions for the two hybridizations, as discussed above. It is clear that the probability of having tensile stress at an $\mathrm{sp}^{2}$ site is largest in the surface area, while it is smaller deeper in the cell. To the contrary, the probability of having compressive stress at an $\mathrm{sp}^{3}$ site is largest in the middle of the slab where bulklike conditions dominate, and smaller at and near the surface. 
The stress probability is related to the position probability shown in Fig. 2. A comparison of the two figures shows that where ever the position probability of fourfold atoms is maximized, the probability for tensile stress is minimized, and vice versa. This again indicates the relationship between bonding and local stress conditions.

\section{DISCUSSION}

The present and previous [5] results show that the bonding hybridization - local stress relationship has a global character. We would further like to comment on two related issues.

It is stated, as a conclusion of thermal annealing experiments [6, 7], that although compressive stress is necessary to sustain the $\mathrm{sp}^{3}$ bonding in ta-C during deposition, it is not so for post-growth processes where stress can be relieved without any appreciable structural changes. This statement is valid when refering to the average intrinsic stress of the film [5]. However, the local atomic stresses do not decrease drastically. They are only re-arranged to yield a zero (small) net stress. In order to sustain the $\mathrm{sp}^{3}$ bonding during growth, and also during annealing, it is necessary to preseve the local compressive conditions.

Since by definition the local atomic stress (hydrostatic pressure) describes the local density fluctuations, it is logical to say that a site under tension reflects a low local density. We know from the above discussion that in such a site will most probably have $\mathrm{sp}^{2}$ bonding, but it is also quite possible for it to have $\mathrm{sp}^{3}$ bonding. This means that there is not an absolute one-to-one correspondence between bonding hybridization and local density. Local densification [4] following ion subplantation (at optimum energies for small relaxation of the density increment) might not actually lead to the formation of an $\mathrm{sp}^{3}$ site. This suggests that the formation of the $\mathrm{sp}^{3}$-rich phase occurs because the rate of densification-compressive stress-sp ${ }^{3}$ bonding events overwhelms the rate of $\mathrm{sp}^{2}$ bonding generation.

\section{CONCLUSIONS}

We examined in this paper the effect of the surface environment on the local atomic stresses, which arise from incompatibilities due to the non-equivalent bonding hybridizations in ta-C. We found a close relationship between hybridization and local stress conditions, as previously found in simulations of the bulk system, and we conclude that this relationship has a global character.

[1] D.R. McKenzie, Rep. Prog. Phys. 59 (1996) 1611.

[2] D.R. McKenzie, D. Muller, and B.A. Pailthorpe, Phys. Rev. Lett. 67 (1991) 773.

[3] C.A. Davis, Thin Solid Films 226 (1993) 30.

[4] J. Robertson, Diam. Relat. Mater. 2 (1993) 984.

[5] P.C. Kelires, Phys. Rev. Lett. 73 (1994) 2460.

[6] T.A. Friedmann, J.P. Sullivan, J.A. Knapp, D.R. Tallant, D.M. Follstaedt, D.L. Medlin, and P.B. Mirkarimi, Appl. Phys. Lett. 71 (1997) 3820.

[7] A.C. Ferrari, B. Kleinsorge, N.A. Morrison, A. Hart, V. Stolojan, and J. Robertson, J. Appl. Phys. 85 (1999) 7191.

[8] J. Tersoff, Phys. Rev. Lett. 61 (1988) 2879.

[9] P.C. Kelires, Phys. Rev. Lett. 68 (1992) 1854.

[10] P.C. Kelires and E. Kaxiras, Phys. Rev. Lett. 78 (1997) 3479.

[11] P.C. Kelires, J. Non-Cryst. Solids 227-230 (1998) 597.

[12] J. Dong and D.A. Drabold, Phys. Rev. B 57 (1998) 15591.

[13] C.A. Davis, G.A.J. Amaratunga, and K.M. Knowles, Phys. Rev. Lett. 80 (1998) 3280. 

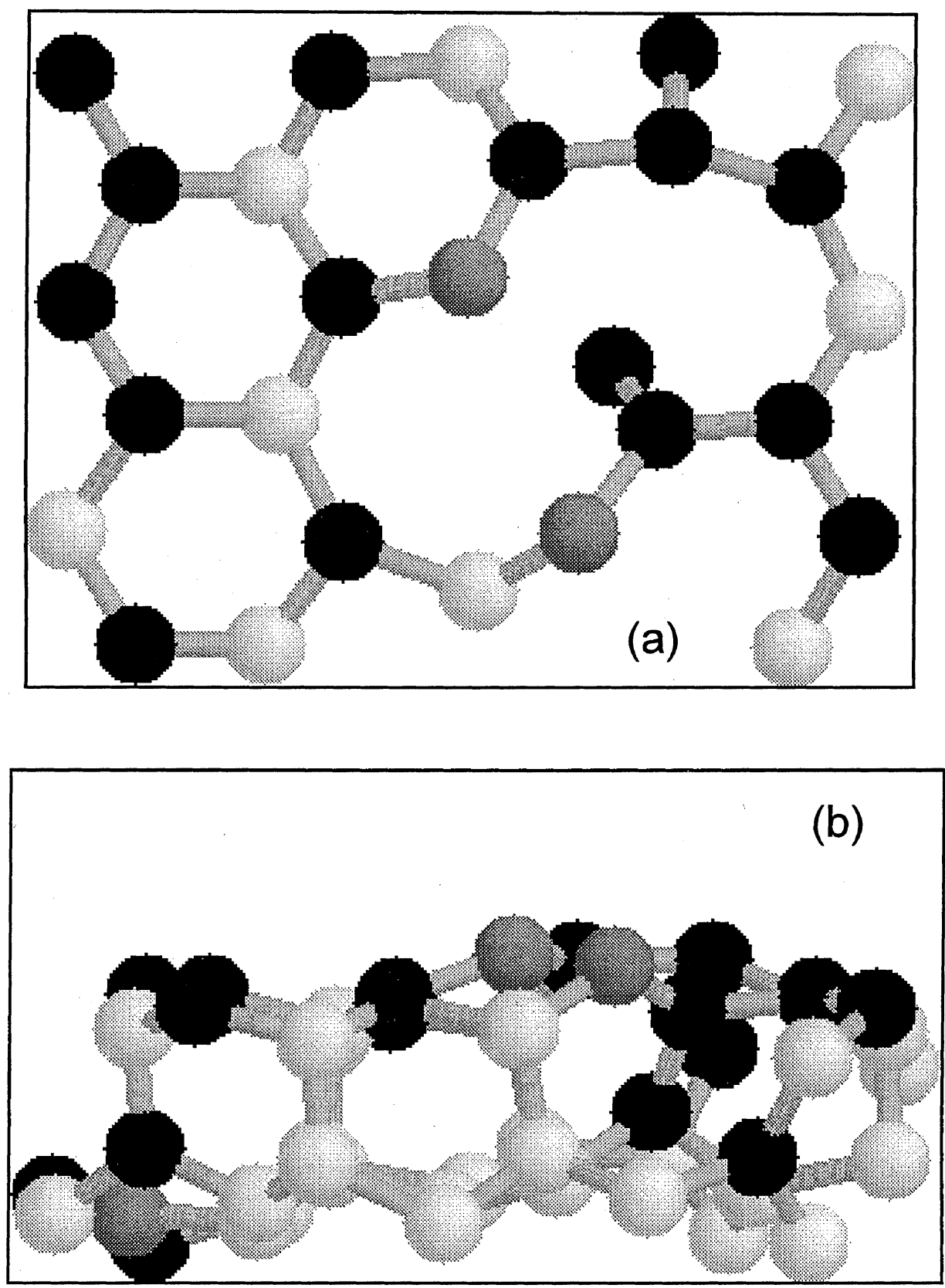

FIG. 1: Ball and stick model of the surface structure of a ta-C network with $\bar{z} \simeq 3.75$. (a) Top view showing the bonding in the surface layer. (b) Side view showing the bonding of surface layer to atoms beneath. Black balls stand for sp ${ }^{2}$ atoms, white balls show $\mathrm{sp}^{3}$ atoms, and grey balls denote twofold atoms. 


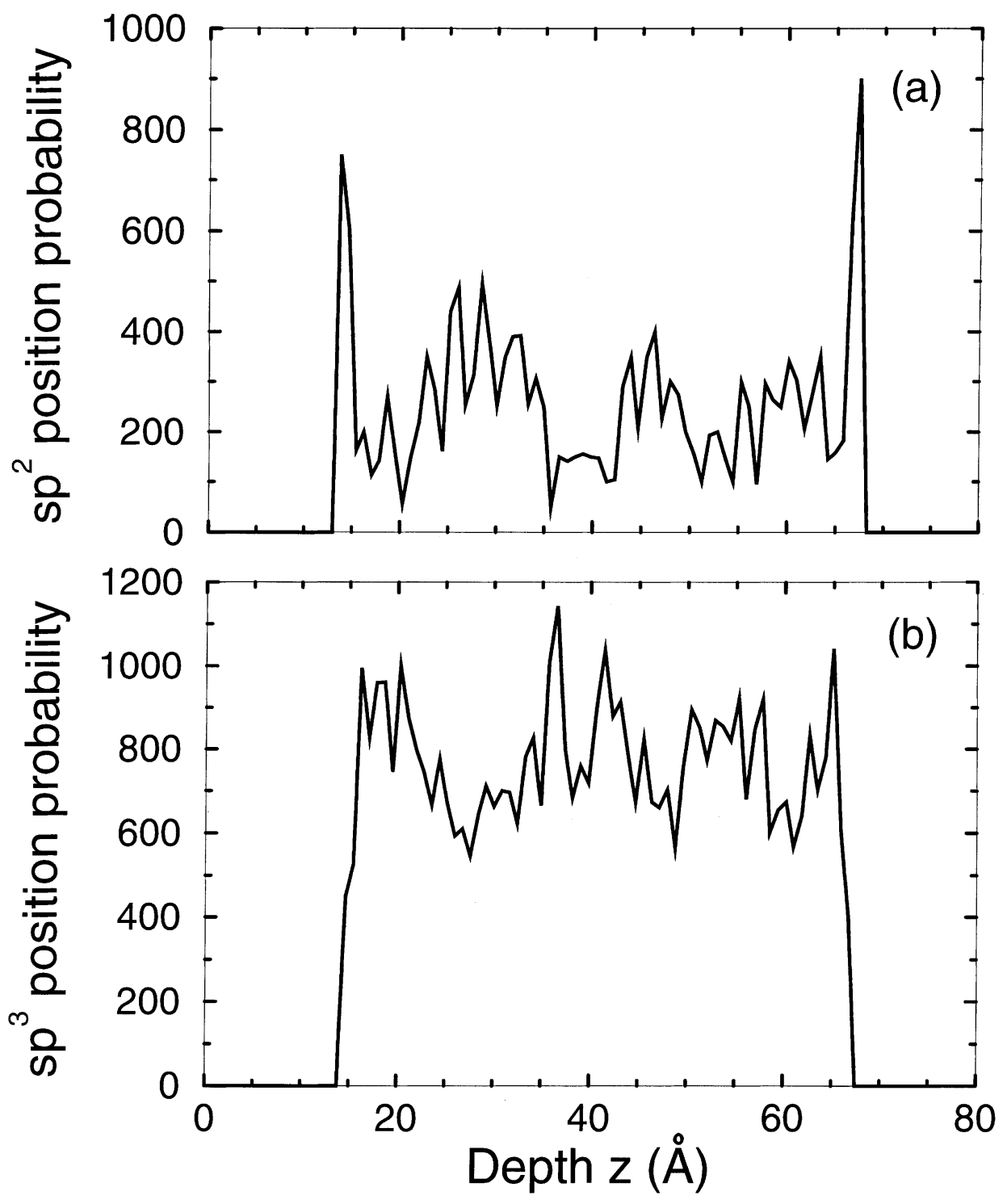

FIG. 2: Position probabilities vs. depth $\mathrm{z}$, at $300 \mathrm{~K}$, in the slab cell of Fig. 1. (a) For $\mathrm{sp}^{2}$ atoms. (b) For $\mathrm{sp}^{3}$ atoms. 


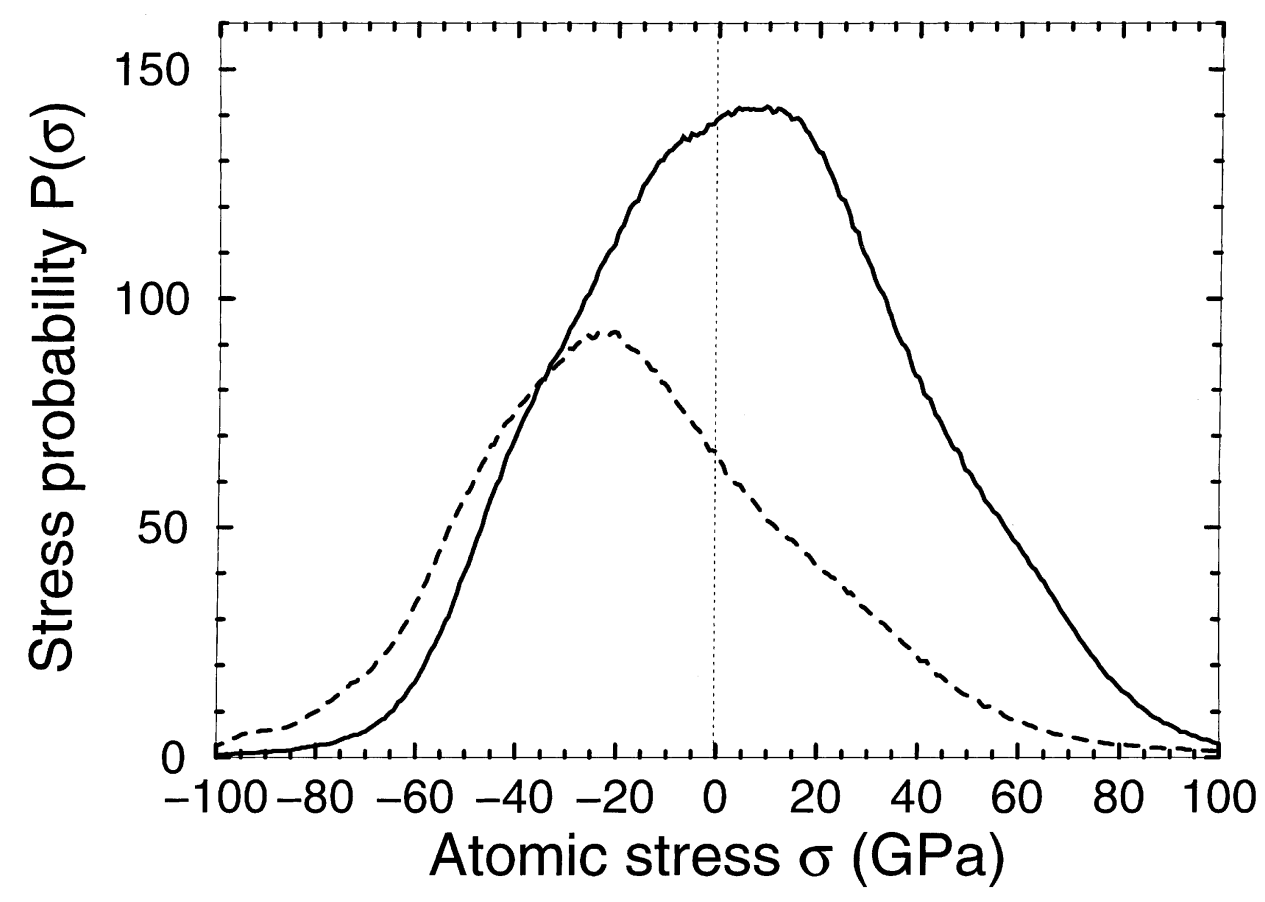

FIG. 3: Distributions of atomic stresses at $300 \mathrm{~K}$ in the slab cell of Fig. 1. Solid and dashed lines are for $\mathrm{sp}^{3}$ and $\mathrm{sp}^{2}$ atoms, respectively. 

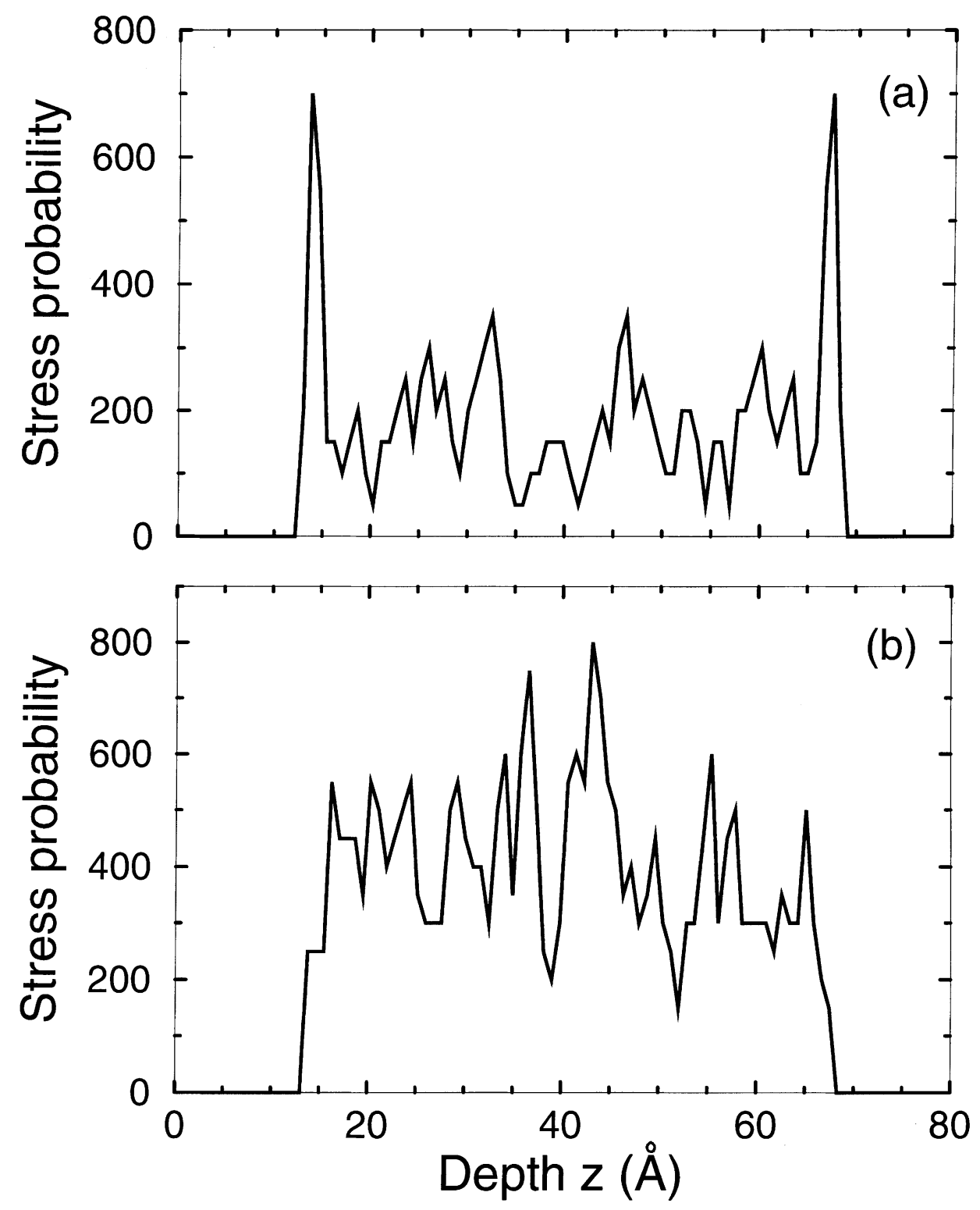

FIG. 4: Stress probability distributions vs. depth z, at $300 \mathrm{~K}$, in the slab cell of Fig. 1. (a) For sp ${ }^{2}$ atoms. (b) For $\mathrm{sp}^{3}$ atoms. 